\title{
Effect of sweep angle and of wall-pressure statistics on the free-field directivity of airfoil trailing-edge noise
}

\author{
Gabriele Grasso* and Michel Roger ${ }^{\dagger}$ \\ Laboratoire de Mécanique des Fluides et d'Acoustique, UMR CNRS 5509, Université de Lyon, \\ École Centrale de Lyon, 69134 Écully CEDEX, France \\ Stéphane Moreau \\ Département de Génie Mécanique, Université de Sherbrooke, Sherbrooke, QC, J1K 2Rl, Canada
}

Highly-swept blades are used in automotive cooling systems, in the fans of modern ultra-high by-pass ratio engines and in counter-rotating open rotors in part in order to reduce noise emission. This work investigates analytically the effect of sweep on the free-field noise emission from the trailing edge of an isolated airfoil, as a first step towards a more complete approach dedicated to rotating blades. Firstly, Amiet-Schwarzschild's technique is extended to the case of a swept airfoil in order to assess the effect of sweep on the sound directivity generated both by a single three-dimensional gust and by the combined effect of all gusts at a given frequency. Sweep also affects the wavenumber contribution of the wall-pressure fluctuations beneath a turbulent boundary layer. This effect is studied by means of a Generalized Corcos model, which allows distribute energy in the chordwise and spanwise wavenumber ranges independently. The sound power level is found proportional to the squared cosine of the sweep angle at all frequencies. Furthermore, the role of the spanwise-wavenumber distribution of wall-pressure power spectral density in amplifying the radiated noise is investigated.

\section{Nomenclature}

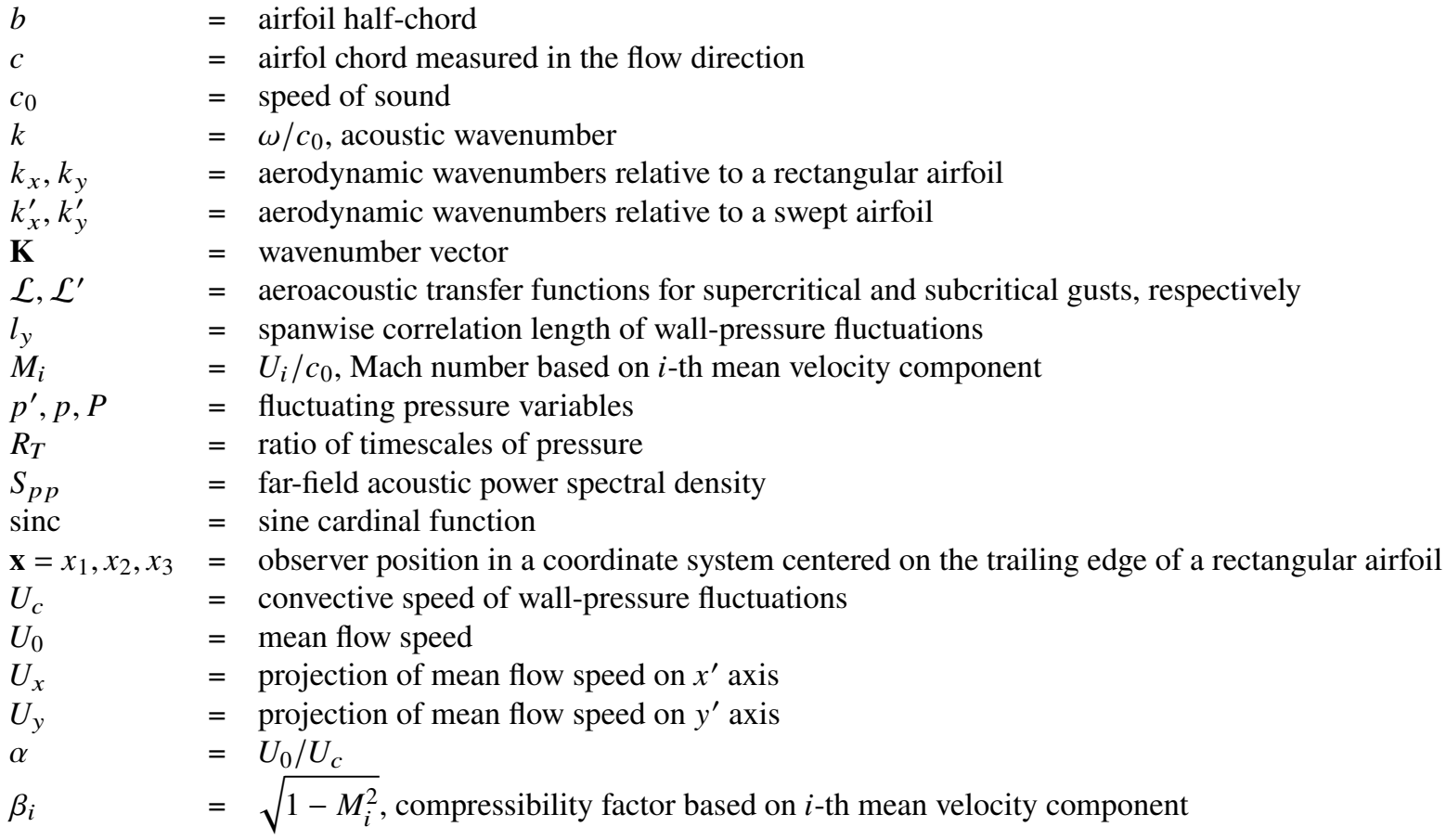

\footnotetext{
*Post-doctoral Researcher, Laboratoire de Mécanique des Fluides et Acoustique, AIAA Member, gabriele.grasso@ec-lyon.fr.

† Professor, Laboratoire de Mécanique des Fluides et Acoustique, AIAA Member.

†Professor, Département de Génie Mécanique, AIAA Lifetime Member.
} 


$\begin{array}{lll}\beta_{C} & =\text { Clauser's parameter } \\ \delta & =\text { boundary layer thickness } \\ \delta^{*} & =\text { boundary layer displacement thickness } \\ \Delta & =\delta / \delta^{*} \\ \Pi & =\text { wake strength parameter } \\ \Pi\left(\omega, k_{x}, k_{y}\right) & =\text { wavenumber-frequency spectral density of wall-pressure fluctuations } \\ \Pi_{0}\left(\omega, k_{y}\right) & =\text { streamwise-integrated wavenumber-frequency spectral density of wall-pressure fluctuations } \\ \rho_{0} & =\text { flow density in a quiescent medium } \\ \tau_{\max } & =\text { maximum shear stress across the boundary layer } \\ \varphi_{p p} & =\text { frequency spectrum of wall-pressure fluctuations } \\ \psi & =\text { sweep angle } \\ \omega & =\text { reduced frequency } \\ \frac{(\cdot)}{} & =\text { normalization by the half-chord, } b\end{array}$

\section{Introduction}

The turbulent boundary layer over an aerodynamic profile generates a spectrum of wall-pressure fluctuations that is scattered as acoustic waves at the trailing edge. In general, trailing-edge noise is the minimum achievable noise of a blade operating in a homogeneous stationary flow. It is therefore of great interest in a variety of industrial applications, typically low-Mach number cooling fans but also ultra-high by-pass ratio (UHBR) engines and counter-rotating open rotors (CROR). In particular, these aero-engine architectures both allow for a substantial reduction of fuel consumption and ongoing research focuses on the mechanisms of their noise emission to further reduce the environmental impact of air transportation. For car-engine cooling fans, sweep is a possible way of improving passenger comfort.

The use of swept blades for the mitigation of several noise generation mechanisms in aeroengine architectures and low-speed ventilation fans has been investigated experimentally and numerically over the years. Vad [1] studied different leading- and/or trailing-edge swept airfoil configurations and concluded that local unloading of the airfoil can be achieved by applying sweep to either edge, irrespectively of the shape of the stacking line, because it is only the component of the mean velocity perpendicular to the edge that contributes to lift generation. Envia et al. [2, 3] investigated the effect of swept and leaned rotor blades and stator vanes in turbofans. De Laborderie and Moreau [4] presented a model of the sound propagated in a duct by a three dimensional outlet guide vane due to wake interaction. Woodward et al. [5] found experimentally that swept vanes significantly reduce the tonal interaction noise emitted by a turbofan. The common conclusion of these studies is that sweep increases the phase changes from hub to tip of the unsteady aerodynamics producing noise thus reducing the source strength (see also [6]). Grasso et al. [7] introduced sweep as a design variable in the AI-driven design of a counter-rotating fan in order to make the most of this effect. Casalino et al. [8] investigated the effect of serrations on swept OGVs, finding that the interaction noise could only be marginally further reduced if the characteristic dimensions of the serrations are sufficiently large compared to the integral scales of the impinging turbulent fluctuations. The role of sweep in the reduction of noise from low-speed axial fans may be more complicated to assess than in the case of turbofans. For instance, in the optimization of a swept benchmark fan carried out by Bamberger and Carolus [9] it was not clear whether the noise reduction was due to sweep or to the reduction of secondary flows. The influence of the operating conditions of the fan on the possibility of reducing noise by means of sweep is highlighted in [10], [11] and [12]. Herold et al. [10] also pointed out that vortex shedding from the trailing-edge of a blade is significantly dependent on the component of the mean flow speed perpendicular to the edge. Therefore, sweep could help reducing this noise generation mechanism as well.

Amiet-Schwarzschild's approach [13-15], originally developed for the case of an airfoil with unswept leading edge, has been later extended to the investigation of turbulence interaction noise from the leading-edge of swept airfoils and blades. The sweep angle was first introduced in the analytical formulation of Amiet's leading-edge theory by Rozenberg [16], followed by Carazo et al. [17, 18] who studied the effect of sweep in reducing CROR wake-interaction noise. The same approach was applied to the study of blade-vortex impingement noise by Roger et al. [19], Giez et al. [20] and Quaglia et al. [21]. The swept-airfoil problem focussed on the assessment of the unsteady lift had already been tackled by Adamczyk [22] using the Wiener-Hopf technique with equivalent results. Concerning the trailing-edge noise modeling, the main scattering formulation obtained by Amiet [23] has been corrected by taking into account a leading-edge back-scattering contribution with a second application of Schwarzschild's technique [24, 25], in order to improve the low-frequency prediction. However, this theory refers only to the case of an edge perpendicular to the mean flow velocity. The scope of this paper is to introduce the sweep angle in Amiet's formulation of trailing-edge 


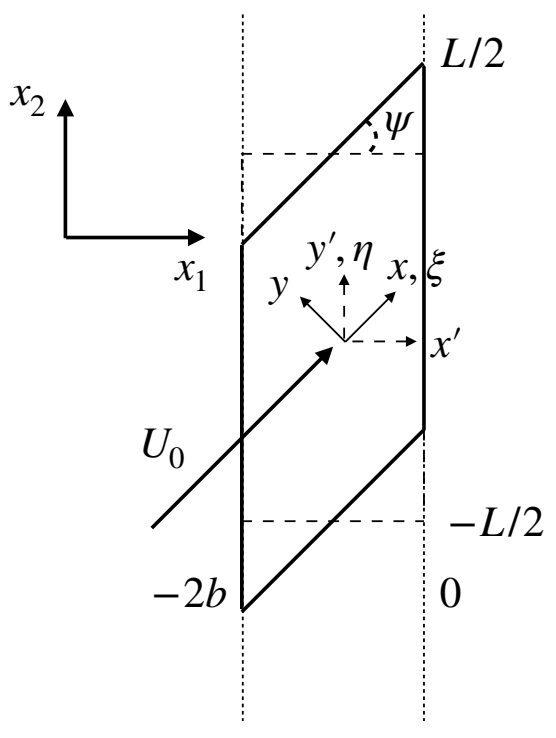

Fig. 1 Reference frames and integration surfaces considered for the formulation of the noise radiated by a swept blade segment (adapted from [17|). The reference frames for integration are displaced at the center of the rectangular integration surface for clarity.

noise. It will be shown that this modification of the original formulation has a twofold effect. On the one hand, sweep modifies the directivity of the noise emitted at a given frequency with respect to the case of a rectangular airfoil, all other conditions being held constant, as was already noticed in the corresponding leading-edge study [17]. On the other hand, the distribution of energy of the incident wall-pressure field is also modified due to the rotation of the reference frame aligned with the edge. It is interesting to investigate this effect as well, because the sound power spectral density (PSD) is directly proportional to that of the incident wall-pressure fluctuations and therefore the quantification of wall-pressure statistics is critical for obtaining an accurate sound prediction.

Various models of the spectrum of wall-pressure fluctuations are available in literature, which are either semiempirical (see [26] for an exhaustive review) or based on the Poisson equation governing the unsteady pressure in an incompressible turbulent boundary layer. Sound predictions obtained with Amiet's theory are very sensitive to the selected wall-pressure spectrum formulation, as shown in [27, 28]. For this reason, the calculations presented in this work are carried out using a model of the statistics of the wall-pressure fluctuations in the wavenumber domain that can be adjusted parametrically, in order to evaluate the effect of different wavenumber-domain distributions on the emitted noise.

\section{Application of Amiet-Schwarzschild's method to the noise emitted by a swept trailing-edge}

\section{A. Derivation of the canonical wave equation for the disturbance pressure}

An analytic expression is sought for the disturbance pressure generated when an incident wall-pressure field is convected past the trailing edge of an airfoil. The disturbance pressure acts as equivalent acoustic sources and the corresponding far-field sound pressure is obtained from Curle's acoustic analogy, yielding an analytical radiation integral. The trailing edge is assimilated to the edge of a half-plane for the application of Schwarzschild's theorem. Only the main trailing-edge scattering term has been derived in this work. The correction for back-scattering from the leading edge could be obtained with a second iteration of Schwarzschild's technique but it is not expected to change qualitatively the directivity of the radiated sound. Furthermore, its effect is significant only at low frequencies (see [25]).

The analytical work presented below follows that of Roger \& Moreau [24] for the case of an unswept airfoil as in Fig. 1] (dashed lines). If the sweep angle $\psi$ defined in Fig. 1] vanishes, the following results coincide with those of [24]. 
The convected wave equation for the disturbance pressure is written as

$$
\nabla^{2} p^{\prime}-\frac{1}{c_{0}^{2}} \frac{\mathrm{D}^{2}}{\mathrm{D} t^{2}} p^{\prime}=0
$$

Due to the application of a sweep angle $\psi$, the mean flow speed has a finite component along the spanwise direction and therefore the total derivative operator has the form

$$
\frac{\mathrm{D}^{2}}{\mathrm{D} t^{2}}=\left(\frac{\partial}{\partial t}+U_{x} \frac{\partial}{\partial x^{\prime}}+U_{y} \frac{\partial}{\partial y^{\prime}}\right)^{2} .
$$

Sinusoidal pressure gusts are characterized by wavenumbers $\left(k_{x}, k_{y}\right)$ in the frame of reference aligned with the mean flow, $(x, y)$ which are transposed in the frame attached to the swept airfoil trailing-edge, $\left(x^{\prime}, y^{\prime}\right)$ with the following transformation:

$$
\left\{\begin{array}{l}
k_{x}^{\prime}=k_{x} \cos \psi-k_{y} \sin \psi \\
k_{y}^{\prime}=k_{x} \sin \psi+k_{y} \cos \psi
\end{array}\right.
$$

The factorization of the disturbance pressure at the reduced frequency $\omega$ as

$$
\begin{aligned}
p^{\prime}\left(x^{\prime}, y^{\prime}, z, t\right) & =P\left(x^{\prime}, y^{\prime}, z\right) \mathrm{e}^{\mathrm{i} \omega t} \\
P\left(x^{\prime}, y^{\prime}, z\right) & =p\left(x^{\prime}, z\right) \mathrm{e}^{\mathrm{i}\left(k_{x}^{\prime} M_{x}^{2} / \beta_{x}^{2}\right) x^{\prime}} \mathrm{e}^{-\mathrm{i} k_{y}^{\prime} y^{\prime}}
\end{aligned}
$$

and using Prandtl-Glauert/Reissner/Lorentz transformation allows to re-write Eq. (1) as a canonical wave equation

$$
\frac{\partial^{2} p}{\partial X^{\prime 2}}+\frac{\partial^{2} p}{\partial Z^{2}}+\kappa^{2} p=0
$$

with the normalized coordinates $X^{\prime}=x^{\prime} / b, Y^{\prime}=y^{\prime} \beta_{x} / b$ and $Z=z \beta_{x} / b$ and the parameter

$$
\kappa^{2}=\mu^{2}-\frac{\bar{k}_{y}^{2}}{\beta_{x}^{2}} \quad \text { with } \quad \mu^{2}=\frac{\bar{k}_{x}^{2} M_{x}^{2}}{\beta_{x}^{4}} .
$$

If $\kappa^{2}>0$, the differential equation is hyperbolic and the gust is said supercritical, whereas if $\kappa<0$, the differential equation is elliptic and the gust is said subcritical. Subcritical gusts are evanescent in the case of an infinite-span airfoil. However, they are considered because the acoustic field is obtained by integrating the effect of all gusts over the actual finite - airfoil surface.

\section{B. Solution of the canonical wave equation}

An incident pressure gust at the frequency $\omega$, convected upstream of the trailing edge with speed $U_{c}=U_{0} / \alpha$, with $\alpha>1$, can be represented as

$$
\begin{aligned}
p_{0}^{\prime}\left(x^{\prime}, y^{\prime}, z, t\right) & =\mathrm{e}^{-\mathrm{i} \alpha k_{x}^{\prime} x^{\prime}} \mathrm{e}^{\mathrm{i} \omega t} \\
P_{0}\left(x^{\prime}, y^{\prime}, z\right) & =\mathrm{e}^{-\mathrm{i} k_{x}^{\prime} x^{\prime}\left(\alpha+M_{x}^{2} / \beta_{x}^{2}\right)} \mathrm{e}^{\mathrm{i} k_{y}^{\prime} y^{\prime} / \beta_{x}} .
\end{aligned}
$$

It is assumed that the wall is perfectly rigid and therefore the normal-to-wall gradient of the corresponding disturbance pressure, $p_{1}^{\prime}$, vanishes on the surface of the half-plane. Furthermore the disturbance pressure is equal and opposite to the incident pressure field at the trailing edge and in the wake according to the Kutta condition. These boundary conditions are expressed as

$$
\begin{cases}\frac{\partial p_{1}}{\partial Z}\left(X^{\prime}, 0\right)=0 & \text { for } \quad X^{\prime}<0 \\ p_{1}\left(X^{\prime}, 0\right)=-\mathrm{e}^{-\mathrm{i} \bar{k}_{x}^{\prime} X^{\prime}\left(\alpha+M_{x}^{2} / \beta_{x}^{2}\right)} \mathrm{e}^{\mathrm{i} \bar{k}_{y}^{\prime} Y^{\prime} / \beta_{x}} & \text { for } \quad X^{\prime} \geq 0 .\end{cases}
$$

Schwarzschild's theorem [15] provides the following solution of the canonical equation (4) for the disturbance pressure with the boundary conditions of Eq. (6) for $X^{\prime}<0$

$$
p_{1}\left(X^{\prime}, 0\right)=-\frac{1}{\pi} \mathrm{e}^{\mathrm{i} \bar{k}_{y}^{\prime} Y^{\prime} / \beta_{x}} \int_{0}^{\infty} \sqrt{-\frac{X^{\prime}}{\zeta}} \frac{\mathrm{e}^{-\mathrm{i} \kappa\left(\zeta-X^{\prime}\right)}}{\zeta-X^{\prime}} \mathrm{e}^{-\mathrm{i} \bar{k}_{x}^{\prime} \zeta\left(\alpha+M_{x}^{2} / \beta_{x}^{2}\right)} \mathrm{d} \zeta
$$


Defining the complex-valued function

$$
\mathrm{E}^{*}(x)=\int_{0}^{x} \frac{\mathrm{e}^{-\mathrm{i} t}}{\sqrt{2 \pi t}} \mathrm{~d} t=\mathrm{C}_{2}(x)-\mathrm{iS}_{2}(x)
$$

where $C_{2}$ and $S_{2}$ are the Fresnel integrals (see [29]), Eq. (7) is re-formulated in the supercritical case $(\kappa>0)$ as

$$
P_{1}^{\text {sup }}\left(X^{\prime}, 0\right)=\mathrm{e}^{-\mathrm{i} \alpha \bar{k}_{x}^{\prime} X^{\prime}}\left[(1+i) \mathrm{E}^{*}\left(-\left(\alpha \bar{k}_{x}^{\prime}+\kappa+\mu M_{x}\right) X^{\prime}\right)-1\right]
$$

If $\psi=0$ and $k_{y}^{\prime}=0$, this corresponds to the original result derived by Amiet in [23].

Equation (7) is applied to the subcritical case $(\kappa<0)$ as well by defining the parameter

$$
\kappa^{\prime 2}=-\kappa^{2}=\frac{\bar{k}_{y}^{\prime 2}}{\beta_{x}^{2}}-\mu^{2}
$$

Although $\kappa^{\prime}$ is a double-valued constant $\left(\kappa^{\prime}= \pm \mathrm{i} \kappa\right)$, it is necessary to select the value $\kappa^{\prime}=-\mathrm{i} \kappa$ to ensure the asymptotic decay to zero at an infinite distance from the source of the scattered pressure field. Defining the complex error function $\Phi^{0}(\sqrt{\mathrm{i} x})=\sqrt{2} \mathrm{e}^{\mathrm{i} \pi / 4} \mathrm{E}^{*}(x)$, the subcritical disturbance pressure solution is expressed as

$$
P_{1}^{\mathrm{sub}}\left(X^{\prime}, 0\right)=\mathrm{e}^{-\mathrm{i} \alpha \bar{k}_{x}^{\prime} X^{\prime}}\left[\Phi^{0}\left(\left[-i\left(\alpha \bar{k}_{x}^{\prime}-\mathrm{i} \kappa^{\prime}+\mu M_{x}\right) X^{\prime}\right]^{1 / 2}\right)-1\right]
$$

\section{Radiation integrals}

The acoustic far-field pressure corresponding to a disturbance pressure gust of wavenumber $\mathbf{K}$, such that $\omega=$ $\mathbf{U}_{\mathbf{0}} \cdot \mathbf{K}=U_{x} k_{x}^{\prime}+U_{y} k_{y}^{\prime}$, is given by Curle [30] as

$$
p_{K}(\mathbf{x}, \omega)=-\frac{\mathrm{i} \omega x_{3}}{4 \pi c_{0} S_{0}^{2}} \int_{S_{y}} \Delta P\left(X^{\prime}, 0\right) \mathrm{e}^{\mathrm{i} \omega R_{t} / c_{0}} \mathrm{e}^{-\mathrm{i} \bar{k}_{y}^{\prime} Y^{\prime}} \mathrm{d} S_{y}
$$

where $\Delta P=2 P_{1}$ is the induced source distribution accounting for the opposite disturbance pressures induced on both sides of the airfoil, acting as an equivalent dipole distribution. The convection of acoustic waves by an external mean flow with velocity components $U_{x}$ and $U_{y}$ along the $x^{\prime}$ and $y^{\prime}$ directions, respectively, is accounted for using the following modified coordinates (see [16]):

$$
\begin{aligned}
& R_{t}=\frac{1}{\beta_{0}^{2}}\left(R_{s}-M_{x}\left(x_{1}-x^{\prime}\right)-M_{y}\left(x_{2}-y^{\prime}\right)\right) \\
& R_{S} \approx S_{0}\left(1-\frac{\left(1-M_{y}^{2}\right) x_{1} x^{\prime}+\left(1-M_{x}^{2}\right) x_{2} y^{\prime}}{S_{0}^{2}}\right)
\end{aligned}
$$

and

$$
S_{0}=\sqrt{\beta_{x}^{2} x_{1}^{2}+\beta_{y}^{2} x_{2}^{2}+\beta_{0}^{2} x_{3}^{2}} .
$$

Equation 10 contains a double integral of the equivalent dipole distribution over the airfoil surface. Considering the shape of the swept airfoil, this integral is solved in the non-Cartesian reference frame $(\xi, \eta)$ represented in Fig. 1 , such that

$$
\left\{\begin{array}{l}
x^{\prime}=\xi \cos \psi \\
y^{\prime}=\eta+\xi \sin \psi
\end{array}\right.
$$

Introducing Eq. (11) in Eq. 10, yields

$$
p_{K}(\mathbf{x}, \omega)=-\frac{\mathrm{i} \omega x_{3} \cos \psi}{2 \pi c_{0} S_{0}^{2}} b^{2} \int_{-\frac{2}{\cos \psi}}^{0} \int_{-\frac{L}{2 b}}^{\frac{L}{2 b}} P_{1}(\bar{\xi}, 0) \mathrm{e}^{\mathrm{i} \omega R_{t} / c_{0}} \mathrm{e}^{-\mathrm{i} \bar{k}_{y}^{\prime}(\bar{\eta}+\bar{\xi} \sin \psi)} \mathrm{d} \bar{\xi} \mathrm{d} \bar{\eta} .
$$


Starting from the supercritical case, defining the parameters

$$
\begin{aligned}
& B=\left(\alpha \bar{k}_{x}^{\prime}+\mu M_{x}+\kappa\right) \cos \psi \\
& C=\alpha \bar{k}_{x}^{\prime} \cos \psi+\bar{k}_{y}^{\prime} \sin \psi-\frac{\bar{k}}{\beta_{0}^{2}}\left(\frac{\beta_{y}^{2} x_{1} \cos \psi}{S_{0}}+\frac{\beta_{x}^{2} x_{2} \sin \psi}{S_{0}}-M_{0}\right)
\end{aligned}
$$

and the complex amplitude of the source distribution

$$
f_{1}^{\text {sup }}(\bar{\xi})=(1+\mathrm{i}) \mathrm{E}^{*}[-B \bar{\xi}]-1
$$

the radiation integral of Eq. (12) becomes

$$
p_{K}(\mathbf{x}, \omega)=-\frac{\mathrm{i} \omega x_{3} \cos \psi}{2 \pi c_{0} S_{0}^{2}} b^{2} \mathrm{e}^{\mathrm{i} k / \beta_{0}^{2}\left(S_{0}-M_{x} x_{1}-M_{y} x_{2}\right)} \int_{-\frac{2}{\cos \psi}}^{0} f_{1}(\bar{\xi}) \mathrm{e}^{-\mathrm{i} C \bar{\xi}} \mathrm{d} \bar{\xi} \int_{-\frac{L}{2 b}}^{\frac{L}{2 b}} \mathrm{e}^{-\mathrm{i} \bar{\eta}\left[\bar{k}_{y}^{\prime}-\bar{k} / \beta_{0}^{2}\left(\beta_{x}^{2} x_{2} / S_{0}-M_{y}\right)\right]} \mathrm{d} \bar{\eta} .
$$

The integral involving $\bar{\eta}$ is independent of the dipole distribution and is solved as

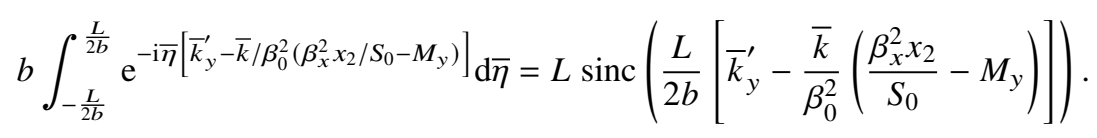

The integral involving $\bar{\xi}$ represents the aeroacoustic transfer function; in the supercritical case this is

$$
\begin{aligned}
\mathcal{L}^{\sup }\left(k_{x}^{\prime}, k_{y}^{\prime}\right) & =\int_{-\frac{2}{\cos \psi}}^{0} f_{1}^{\text {sup }}(\bar{\xi}) \mathrm{e}^{-\mathrm{i} C \bar{\xi}} \mathrm{d} \bar{\xi} \\
& =-\frac{\mathrm{e}^{2 \mathrm{i} C / \cos \psi}}{\mathrm{i} C}\left\{(1+\mathrm{i}) \mathrm{e}^{-2 \mathrm{i} C / \cos \psi} \sqrt{\frac{2 B}{\cos \psi}} \mathrm{ES}^{*}\left(\frac{2(B-C)}{\cos \psi}\right)-(1+\mathrm{i}) \mathrm{E}^{*}\left(\frac{2 B}{\cos \psi}\right)+1-\mathrm{e}^{-2 \mathrm{i} C / \cos \psi}\right\}
\end{aligned}
$$

where ES* is the modified Fresnel integral introduced in [31] as

$$
\operatorname{ES}^{*}(z)=\frac{\mathrm{E}^{*}(z)}{\sqrt{z}}=\frac{1-\mathrm{i}}{2} \frac{\Phi^{0}(\sqrt{\mathrm{i} z})}{\sqrt{z}}
$$

In the subcritical case, defining the parameter

$$
A_{1}^{\prime}=\left(\alpha \bar{k}_{x}^{\prime}+\mu M_{x}-\mathrm{i} \kappa^{\prime}\right) \cos \psi
$$

and the complex amplitude of the source distribution

$$
f_{1}^{\text {sub }}(\bar{\xi})=\Phi^{0}\left(\left[-\mathrm{i} A_{1}^{\prime} \bar{\xi}\right]^{1 / 2}\right)-1
$$

yields the following aeroacoustic transfer function

$$
\begin{aligned}
\mathcal{L}^{\mathrm{sub}}\left(k_{x}^{\prime}, k_{y}^{\prime}\right) & =\int_{-\frac{2}{\cos \psi}}^{0} f_{1}^{\mathrm{sub}}(\bar{\xi}) \mathrm{e}^{-\mathrm{i} C \bar{\xi}} \mathrm{d} \bar{\xi} \\
& =-\frac{\mathrm{e}^{2 \mathrm{i} C / \cos \psi}}{\mathrm{i} C}\left\{(1+\mathrm{i}) \sqrt{\frac{2 A_{1}^{\prime}}{\cos \psi}} \mathrm{ES}^{*}\left(\frac{2\left(A_{1}^{\prime}-C\right)}{\cos \psi}\right) \mathrm{e}^{-2 \mathrm{i} C / \cos \psi}-\Phi^{0}\left(\left[\frac{2 \mathrm{i} A_{1}^{\prime}}{\cos \psi}\right]^{1 / 2}\right)+1-\mathrm{e}^{-2 \mathrm{i} C / \cos \psi}\right\} .
\end{aligned}
$$

It can be noticed that both Eqs. (15) and (16) contain the term $-\mathrm{e}^{-2 \mathrm{iC} / \cos \psi}$. According to Amiet [32], the equivalent of this term for the unswept edge cancels out the contribution of the incident pressure $P_{0}$ to the sound radiation from the trailing edge. Therefore, this term will be suppressed in the following noise calculations in order to take into account the effect of both the incident and disturbance pressures. 


\section{Far-field sound power spectral density}

The power spectral density (PSD) of the acoustic pressure radiated at a given frequency $\omega$ results from the integrated contribution of all skewed gusts. Assuming Taylor's hypothesis [33] of frozen convection of the turbulent structures with velocity $U_{c}$, in the case of an unswept trailing edge the only streamwise aerodynamic wavenumber contributing to the frequency $\omega$ is $K_{c}=\omega / U_{c}$. However, a swept trailing edge generates a coupling between the streamwise and spanwise aerodynamic wavenumbers whereby a continuous array of streamwise wavenumbers contributes to the PSD at frequency $\omega$, given by

$$
K_{c}^{\prime}=\frac{\omega}{U_{c} \cos \psi}-k_{y}^{\prime} \tan \psi
$$

Therefore, the PSD observed in the far-field at the position $\mathbf{x}$ and at the frequency $\omega$ results from an integration over all spanwise wavenumbers $k_{y}^{\prime}$ :

$$
S_{p p}(\mathbf{x}, \omega)=\left(\frac{\omega x_{3} \cos \psi L b}{2 \pi c_{0} S_{0}^{2}}\right)^{2} \frac{1}{b} \int_{-\infty}^{+\infty} \Pi_{0}\left(\omega, \bar{k}_{y}^{\prime}\right) \operatorname{sinc}^{2}\left\{\frac{L}{2 b}\left[\bar{k}_{y}^{\prime}-\frac{\bar{k}}{\beta_{0}^{2}}\left(\frac{\beta_{x}^{2} x_{2}}{S_{0}}-M_{y}\right)\right]\right\}\left|\mathcal{L}\left(\bar{K}_{c}^{\prime}, \bar{k}_{y}^{\prime}\right)\right|^{2} \mathrm{~d} \bar{k}_{y}^{\prime} .
$$

The asymptotic limit for large aspect ratio, $L / 2 b$, of the sine cardinal squared is a Dirac delta funtion:

$$
\lim _{L / 2 b \rightarrow+\infty} \operatorname{sinc}^{2}\left\{\frac{L}{2 b}\left[\bar{k}_{y}^{\prime}-\frac{\bar{k}}{\beta_{0}^{2}}\left(\frac{\beta_{x}^{2} x_{2}}{S_{0}}-M_{y}\right)\right]\right\}=\frac{2 \pi b}{L} \delta\left(\bar{k}_{y}^{\prime}-\frac{\bar{k}}{\beta_{0}^{2}}\left(\frac{\beta_{x}^{2} x_{2}}{S_{0}}-M_{y}\right)\right) .
$$

For a large enough aspect ratio, Eq. [18) can be simplified by introducing Eq. [19], as discussed in [24]. The resulting sound PSD formulation is

$$
S_{p p}(\mathbf{x}, \omega)=\left(\frac{\omega x_{3} \cos \psi b}{c_{0} S_{0}^{2}}\right)^{2} \frac{L}{2 \pi} \Pi_{0}\left(\omega, \bar{K}_{y}^{\prime}\right)\left|\mathcal{L}\left(\bar{K}_{c}^{\prime}, \bar{K}_{y}^{\prime}\right)\right|^{2}
$$

with

$$
\bar{K}_{y}^{\prime}=\frac{\bar{k}}{\beta_{0}^{2}}\left(\frac{\beta_{x}^{2} x_{2}}{S_{0}}-M_{y}\right) .
$$

The effect of the large-span simplification is to select a privileged oblique gust for each $x_{2}$ position. In this case, the observer hears only that gust which produces an acoustic wavefront normal to the line joining airfoil and observer [13]. It can also be noticed that the sound PSD is proportional to the squared cosine of the sweep angle: the same conclusion was reached by Ffowcs Williams and Hall [34] by different means.

The closure of Eqs. (18) and (20) is obtained by defining the energy of the incident wall-pressure fluctuations at frequency $\omega$ and spanwise wavenumber $k_{y}^{\prime}$ as

$$
\Pi_{0}\left(\omega, k_{y}^{\prime}\right)=\int_{-\infty}^{+\infty} \Pi\left(k_{x}^{\prime}, k_{y}^{\prime}, \omega\right) \mathrm{d} k_{x}^{\prime}=\frac{1}{\pi} \varphi_{p p}(\omega) l_{y}\left(\omega / U_{c}, k_{y}^{\prime}\right) .
$$

The frequency-wavenumber spectrum is a quantity that could be provided by Direct Numerical Simulation, but for preliminary acoustic evaluation empirical or semi-analytic models are often used, as will be detailed in the next section.

Regardless of the selected model of wall-pressure statistics, the radiation integrals presented in this section allow for an evaluation of how the sweep angle modifies the directivity patterns of the noise emitted by the interaction of the trailing edge with a given three-dimensional incident pressure gust. Two airfoils are taken into consideration for this purpose: a rectangular unswept airfoil and a parallelogram-shaped airfoil with sweep angle $\psi=15^{\circ}$, having the same aspect ratio. Figure 2 compares the directivity lobes of the two airfoils subject to the same incident pressure gusts. Figure 2(a) represents the interaction of the airfoils with a gust parallel to the trailing-edge of the rectangular unswept airfoil, which produces a directivity pattern symmetric with respect to the direction normal to the airfoil surface, typical of a parallel gust interaction. The application of a sweep angle means that the same gust is skewed with respect to the swept airfoil and therefore the directivity pattern is asymmetric because the finite component of the phase velocity of the gust along the airfoil edge sets a privileged direction for the radiation. The effect of the interaction of the two airfoils with a gust parallel to the trailing-edge of the swept airfoil is presented in Fig. 2(b). In this case, the gust is skewed with respect to the unswept airfoil, but the directivity pattern produced by the swept airfoil is asymmetric with respect to the normal-to-wall direction as well. This is due to the asymmetry of the surface of the swept airfoil itself. These results are consistent with those presented in [17] concerning leading-edge noise directivity. 


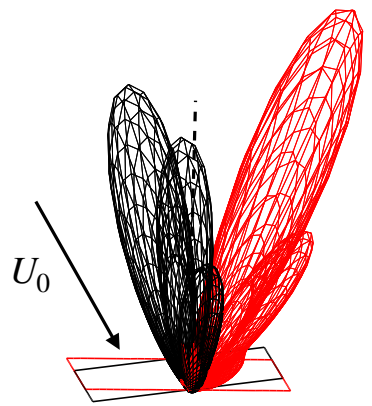

(a) Incident gust parallel to rectangular airfoil

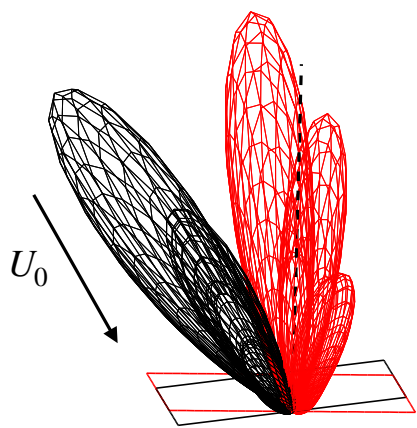

(b) Incident gust parallel to swept airfoil

Fig. 2 Directivity lobes for an unswept rectangular airfoil (black) and a swept parallelogram shaped airfoil (red), interacting with a sinusoidal gust. Observer on a half-sphere, arbitrary units. $M=0.47, \mathrm{kc}=8$, sweep angle $\psi=15^{\circ}$, aspect ratio $\mathrm{L} / \mathrm{c}=\mathbf{1 . 5}$.

\section{Wavenumber-frequency spectral density of wall-pressure fluctuations}

\section{A. Generalized Corcos' model}

A model of the wall-pressure statistics widely applied in literature is Corcos' [35]. This model owes its popularity to some mathematical advantages, namely the separation of the space/wavenumber variables and the capability to go easily from the space/time to the wavenumber/frequency domain. It can be formulated in the following way:

$$
\Pi\left(k_{x}, k_{y}, \omega\right)=\varphi_{p p}(\omega) \frac{\frac{1}{\pi \alpha_{\omega}}}{1+\left(\frac{k_{x}-k_{\omega}}{\alpha_{\omega}}\right)^{2}} \frac{\frac{1}{\pi \beta_{\omega}}}{1+\left(\frac{k_{y}}{\beta_{\omega}}\right)^{2}}
$$

with $\alpha_{\omega}=\alpha_{x} \omega / U_{c}$ and $\beta_{\omega}=\alpha_{y} \omega / U_{c}$. The constants $\alpha_{x}=0.10$ and $\alpha_{y}=0.77$ are within the typical range for smooth rigid walls (see [36]). However, this model is also known to overestimate the contribution of the subconvective streamwise wavenumber range to a given reduced frequency, $\omega$, by as much as $20 \mathrm{~dB}$. A generalization of Corcos' model that corrects the low-wavenumber behavior while preserving the mathematical advantages has been proposed by Caiazzo et al. [36]. It relies on the fact that the wavenumber energy distribution of Corcos' model is represented by a Lorentzian function in each dimension and that a Lorentzian function can be generalized as the magnitude squared of a Butterworth filter of order one. Therefore, the Generalized Corcos' model represents the wavenumber energy distribution with Butterworth filters of arbitrary orders:

$$
\Pi\left(k_{x}, k_{y}, \omega\right)=\varphi_{p p}(\omega) \frac{\frac{n \sin (\pi / 2 n)}{\pi \alpha_{\omega}}}{1+\left(\frac{k_{x}-k_{\omega}}{\alpha_{\omega}}\right)^{2 n}} \frac{\frac{m \sin (\pi / 2 m)}{\pi \beta_{\omega}}}{1+\left(\frac{k_{y}}{\beta_{\omega}}\right)^{2 m}} .
$$

The parameters $m$ and $n$ can be varied independently to obtain the desired wavenumber distribution of wall-pressure fluctuations. It was found in [36] that increasing the order of the streamwise wavenumber filter, $n$, brings the spectral density level closer to experimental evidence in the subconvective range. Yet, the Generalized model does not overcome the main limitation of Corcos. As Singer [37] pointed out, the separation of space/wavenumber variables prevents from representing correctly the wall-pressure correlation in directions that are neither parallel nor normal to the mean flow. The Generalized model is retained in this work because it allows to verify the effect of different wavenumber wall-pressure spectrum distributions on the noise radiated from a trailing edge by simply varying the $m$ and $n$ parameters. For this purpose, the rotation of the wavenumber plane expressed in Eq. (3) is introduced in Eq. 23) obtaining

$$
\Pi\left(k_{x}^{\prime}, k_{y}^{\prime}, \omega\right)=\varphi_{p p}(\omega) \frac{\frac{n \sin (\pi / 2 n)}{\pi \alpha_{\omega}}}{1+\left(\frac{k_{x}^{\prime} \cos \psi+k_{y}^{\prime} \sin \psi-k_{\omega}}{\alpha_{\omega}}\right)^{2 n}} \frac{\frac{m \sin (\pi / 2 m)}{\pi \beta_{\omega}}}{1+\left(\frac{-k_{x}^{\prime} \sin \psi+k_{y}^{\prime} \cos \psi}{\beta_{\omega}}\right)^{2 m}} .
$$


Finally, Rozenberg's model [38] of the frequency wall-pressure PSD, $\varphi_{p p}(\omega)$, is selected for the closure of Eq. 24]. This is an empirical model that takes into account the effects of Reynolds number and adverse pressure gradient, validated on a range of pipe flow and airfoil applications. The functional expression is

$$
\frac{\varphi_{p p}(\omega) U_{0}}{\tau_{\text {max }}^{2} \delta^{*}}=\frac{\left[2.82 \Delta^{2}\left(6.13 \Delta^{-0.75}+F_{1}\right)^{A 1}\right]\left[4.2\left(\frac{\Pi}{\Delta}+1\right)\right] \tilde{\omega}^{2}}{\left[4.76 \tilde{\omega}^{0.75}+F_{1}\right]^{A 1}+\left[C_{3}^{\prime} \tilde{\omega}\right]^{A 2}}
$$

with $\tilde{\omega}=\omega \delta^{*} / U_{0}, A_{1}=3.7+1.5 \beta_{C}, A_{2}=\min \left(3,19 / \sqrt{R_{T}}\right)+4, C_{3}^{\prime}=8.8 R_{T}^{-0.57}$ and

$$
F_{1}=4.76\left(\frac{1.4}{\Delta}\right)^{0.75}\left[0.375 A_{1}-1\right] \text {. }
$$

Note that the typographical error in the definition of $A_{2}$ in [38] has been corrected.

\section{Application of the swept trailing-edge theory to the computation of wall-pressure statistics and sound emission}

\section{A. Application case: controlled-diffusion airfoil}

The numerical tests presented in this section refer to an application case analyzed in [38]: that of a controlled-diffusion airfoil of chord $c=0.1356 \mathrm{~m}$ with a strongly adverse pressure gradient. Reference measurements have been performed in the anechoic room of ECL by Moreau \& Roger [39] and computations have been performed by Christophe et al. [40] for instance. The latter RANS simulations have been selected.The wall-pressure spectrum and the boundary-layer parameters needed for the application of Eq. 25) are collected at a location on the suction side, close to the trailing edge, where the flow is on the verge of separation. The data, presented in Tab. 1 of [38], are $U_{0}=16.9 \mathrm{~m} / \mathrm{s}, \Delta=2.23$, $\tau_{\max }=0.167 \mathrm{~Pa}, \beta_{C}=20.9, \Pi=8.18$ and $\alpha=1.43$. The conditions of this application case are used to test the effect of the introduction of a trailing-edge sweep on the description of the wall-pressure statistics and on the corresponding sound level and directivity.

\section{B. Effect of sweep angle on the wavenumber spectral density of wall-pressure fluctuations}

Examples of wavenumber spectral density prescribed by Eq. [24), normalized by $\varphi_{p p}$, are depicted in Fig. 3 . The left column represents cases in which the mean flow direction is aligned with the $x^{\prime}$ axis. It can be noticed that the local maxima for each $\bar{k}_{y}^{\prime}$ correspond to a line of constant $\bar{k}_{x}^{\prime}=\bar{K}_{c}=\omega b / U_{c}$. The increase of the order of the streamwise Butterworth filter, $n$, causes a much faster decay of the wavenumber spectral density along the $\bar{k}_{x}^{\prime}$ axis. The increase of $m$ has also the effect of flattening the spectral density around the maximum along the $\bar{k}_{y}^{\prime}$ axis. The effect of sweep is introduced in the right column of Fig. 3 for the same $(m, n)$ values. In this case, the line of local maxima is a function of $\bar{k}_{y}^{\prime}$ expressed by Eq. (17). Also, the absolute maximum of the distribution corresponds to a $\bar{k}_{y}^{\prime} \neq 0$ consistently with Eq. (24). The dashdotted lines in each plot represent the limit between subcritical and supercritical gusts according to Eq. (5). For $\psi=0$ the angle of the supercritical range corresponds to $\operatorname{atan}\left(M_{0} / \beta_{0}\right)$, whereas for $\psi \neq 0$ it is slightly smaller, corresponding to atan $\left(M_{x} / \beta_{x}\right)$. However, due to the rotation of the wavenumber plane, more energy enters the supercritical range, especially for $m>1$. These considerations are consistent with those presented by Roger et al. [19] concerning the wavenumber energy distribution of turbulence impinging an airfoil leading edge.

The information needed for noise prediction is the integral of the wavenumber spectral density of wall-pressure fluctuations with respect to the streamwise wavenumber $k_{x}^{\prime}$. This corresponds to the spanwise correlation length of wall-pressure fluctuations, $l_{y}$, according to Eq. (21). It is evident from Eq. (23) that the value of this integral is independent of $n$ if and only if $\psi=0$. On the contrary, the value of $n$ is expected to play a role when $\psi \neq 0$, according to Eq. 24]. Figure 4 represents the variation of $l_{y}$ with the filter orders $(m, n)$ with and without sweep. In both cases, the increase of $m$ flattens the curve around the maximum, as already pointed out, while causing a faster decay of the correlation away from the maximum. The effect of increasing $n$, visible only for $\psi \neq 0$, is to increase slightly the correlation peak for a given $m$. However, there is only a negligible difference between the curves at $n=2$ and $n=3$.

\section{Effect of sweep on far-field sound PSD and directivity}

The effect of sweep on far-field sound PSD is evaluated in Fig. 5 5 using the original Corcos' model (i.e. $m=1, n=1$ in Eq. (24). The effect of the variation of the filter orders will be evaluated in the next section. The left-hand-side plot 


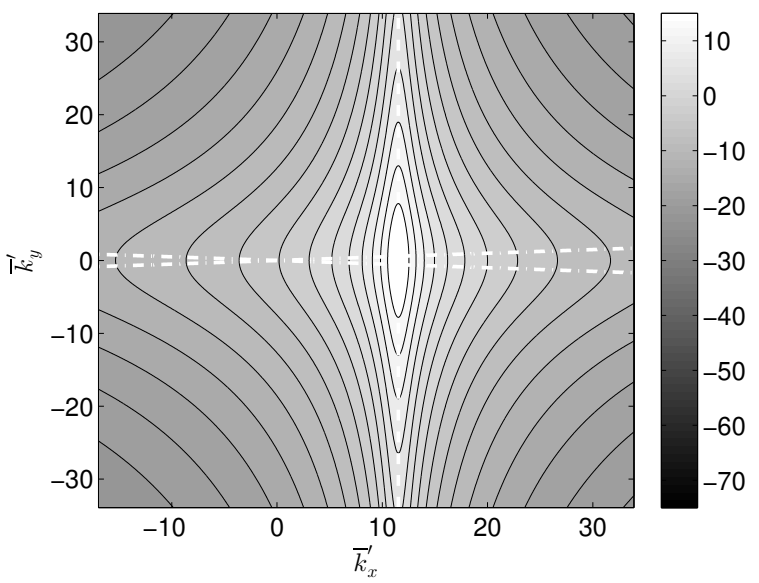

(a) $m=1, n=1, \psi=0$

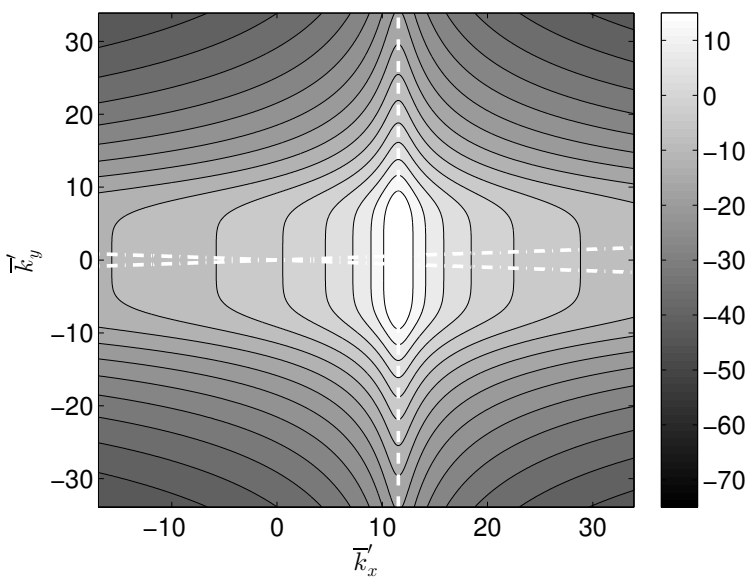

(c) $m=3, n=1, \psi=0$

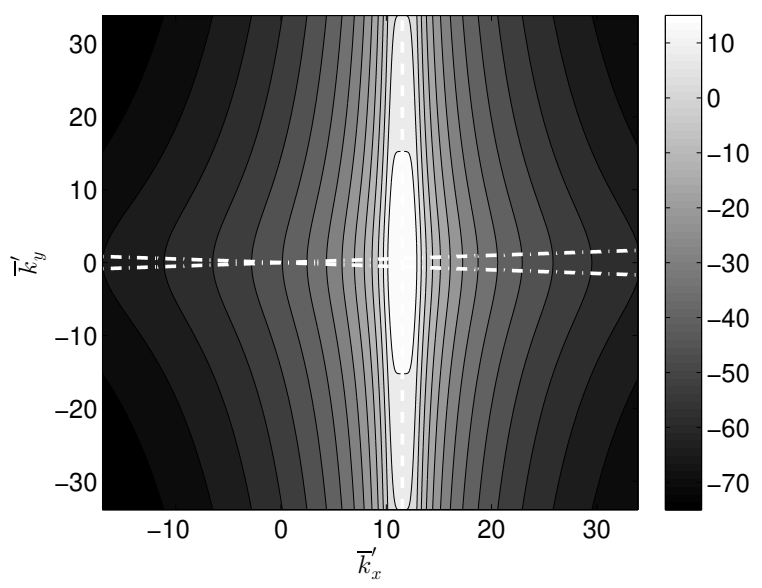

(e) $m=1, n=3, \psi=0$

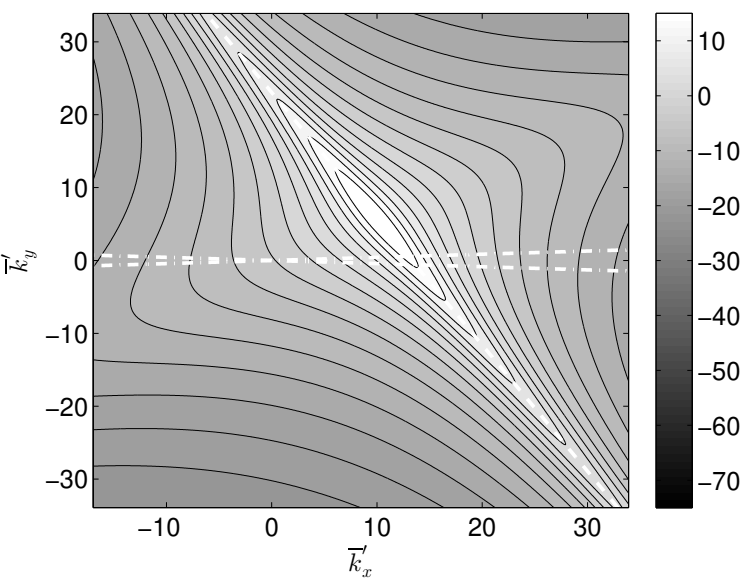

(b) $m=1, n=1, \psi=30^{\circ}$

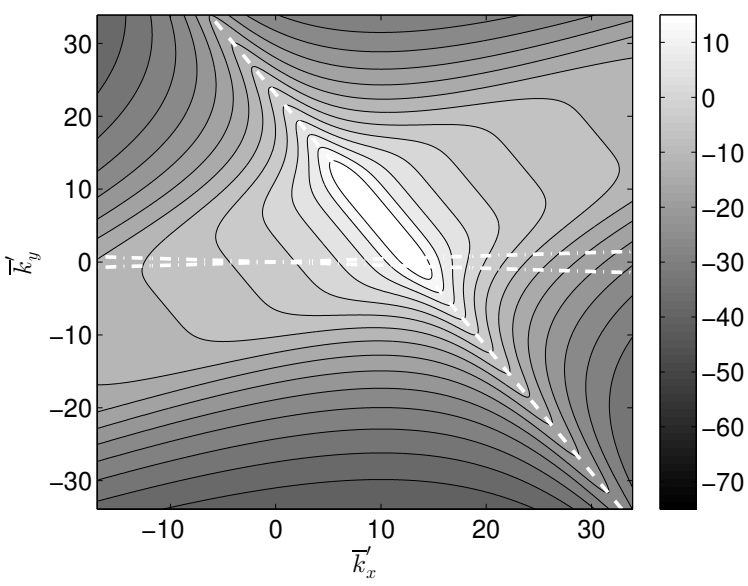

(d) $m=3, n=1, \psi=30^{\circ}$

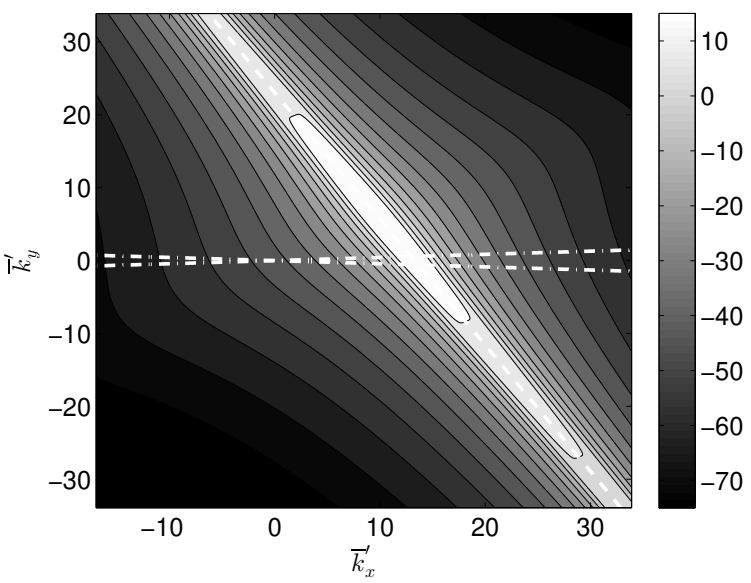

(f) $m=1, n=3, \psi=30^{\circ}$

Fig. 3 Normalized wavenumber wall-pressure spectral density at $f=320 \mathrm{~Hz}$. Left column: $\psi=0$. Right column: $\psi=30^{\circ}$. Dashed line: locus of local maxima corresponding to $K_{c}^{\prime}$. Dash-dotted line: critical limit. 


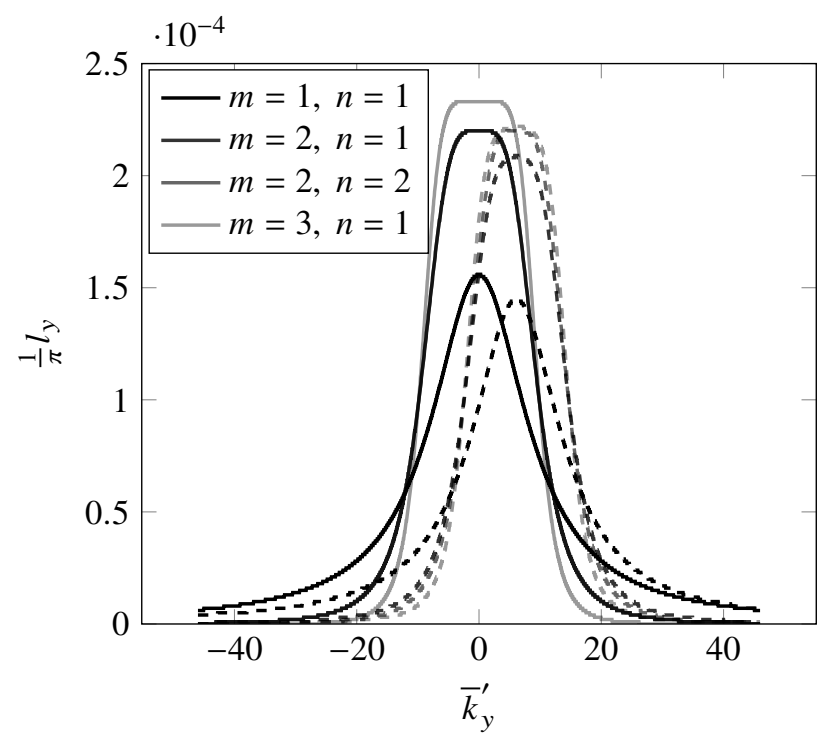

$\cdot 10^{-4}$

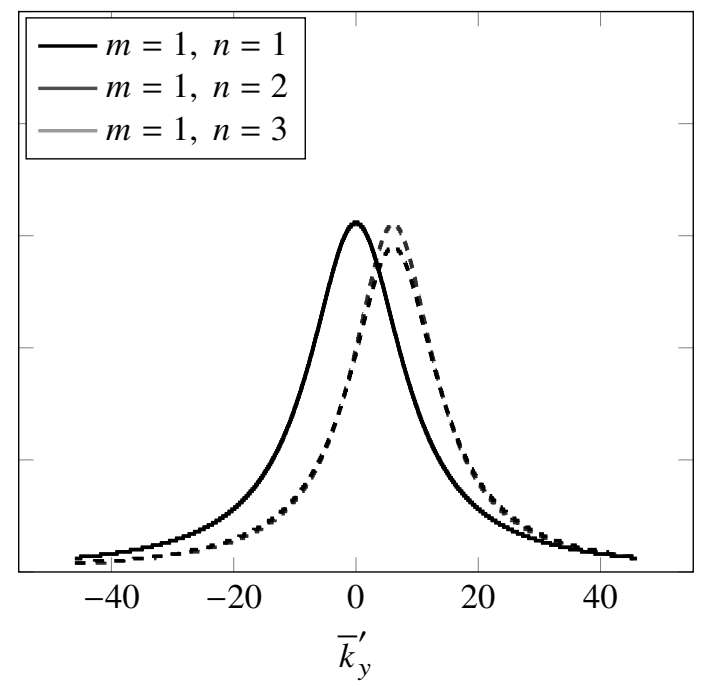

Fig. 4 Spanwise correlation length of wall-pressure fluctuations at $f=320 \mathrm{~Hz}$. Plain lines: $\psi=0$. Dashed lines: $\psi=30^{\circ}$.

depicts the wall-pressure PSD, $\varphi_{p p}(\omega)$, computed with Rozenberg's formulation of Eq. 25) (which agrees very well with the measurements as shown in Fig. 17(e) of [38]). The right-hand-side plot of Fig. 5 compares firstly the sound PSD predicted for an unswept airfoil with and without the correction for leading-edge backscattering. The corrected spectrum corresponds to that depicted in Fig. 18 of [38], which also agrees well with experimental data. The spectrum computed using the main trailing-edge scattering term shows a small discrepancy with respect to the corrected spectrum only at low frequencies. Therefore the lack of a leading-edge backscattering correction in the swept airfoil formulation does not qualitatively alter the results of the noise prediction. This numerical test shows that the increase of $\psi$ causes an overall decrease of the sound PSD in the midspan plane and a shift towards lower frequencies of the dips and humps of interferences in the non-compact frequency range.

Figure 6 represents the directivity of far-field noise calculated in the same conditions on the upper half-sphere around the airfoil. At $f=5 \mathrm{kHz}$, the increase of sweep causes the decrease of the radiated sound power at every angle of observation and a slightly more marked asymmetry of the directivity pattern. At $f=10 \mathrm{kHz}$, the increase of the asymmetry of the directivity with the sweep angle is more marked, to the point that at some observation angles the sound power is higher for higher values of $\psi$.

\section{Effect of wall-pressure wavenumber spectral distribution on sound PSD and directivity}

The effect of the order of the Butterworth filters of the Generalized Corcos' model is investigated in Figs. 7 and 8 Figure 7 shows that in the midspan plane the main effect is that of the parameter $m$, which can raise the sound PSD by approximately $3 \mathrm{~dB}$ increasing from 1 to 2 as can be seen in the left-hand-side plot for $\psi=0$. Increasing $m$ to 3 provides only a further marginal increase of the sound PSD. The effect of $n$, on the contrary, is negligible in the midspan plane for $\psi=30^{\circ}$.

The effect of the filter orders $(m, n)$ on the directivity is shown in Fig. 8 at $f=10 \mathrm{kHz}$. In case of $\psi=0$, the directivity pattern is not qualitatively changed by the increase of $m$, but the amplitude of the radiated sound power increases at all angles of observation. The directivity patterns for the $\psi=30^{\circ}$ case confirm that the main effect is that of the parameter $m$. However, a smaller increase of the sound power at a given $m$ can be observed at positions closer to the airfoil plane for an increase of $n$.

\section{Conclusions}

The analytic considerations and preliminary numerical tests presented in this paper have highlighted the importance of taking into account sweep in a theory of trailing-edge noise. They provide more complete physical insight into 

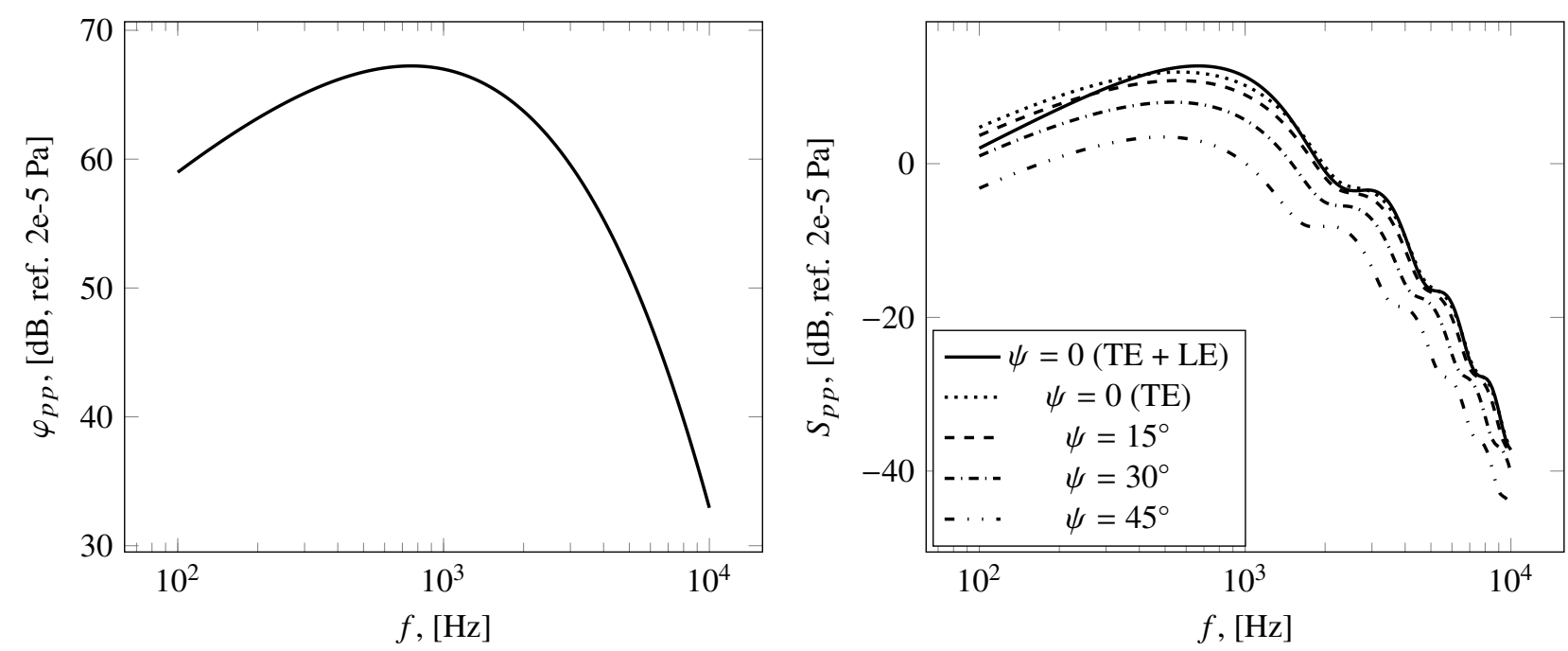

Fig. 5 Left: wall-pressure PSD calculated with Rozenberg's model. Right: effect of sweep on the sound PSD in the midspan plane $\left(x_{2}=0\right)$ at a distance $R=2 \mathrm{~m}$ from the trailing edge.

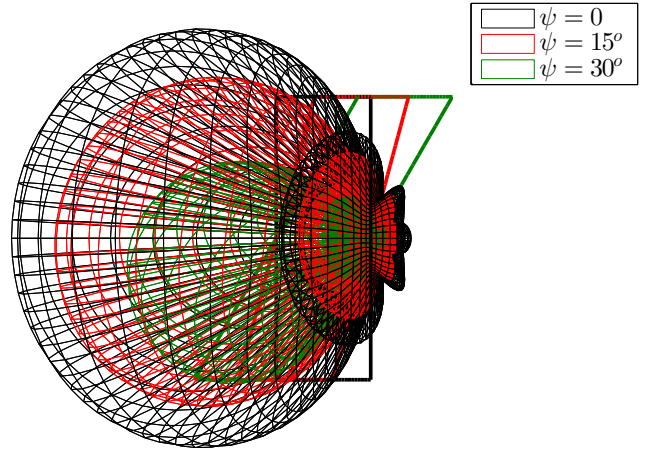

(a) $f=5 \mathrm{kHz}$

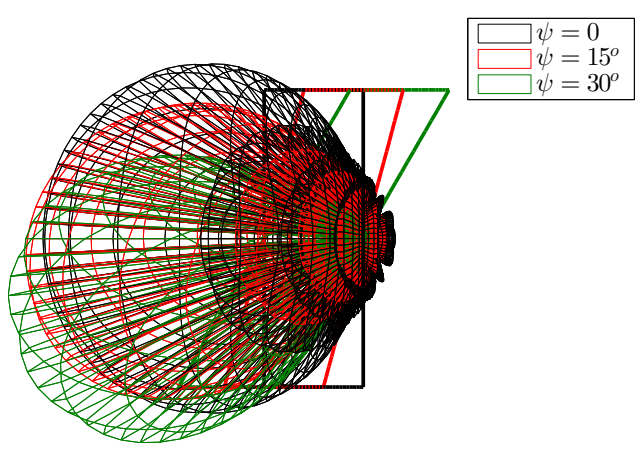

(b) $f=10 \mathrm{kHz}$

Fig. 6 Effect of sweep on the predicted directivity of the noise of the CD airfoil on a half-sphere. 

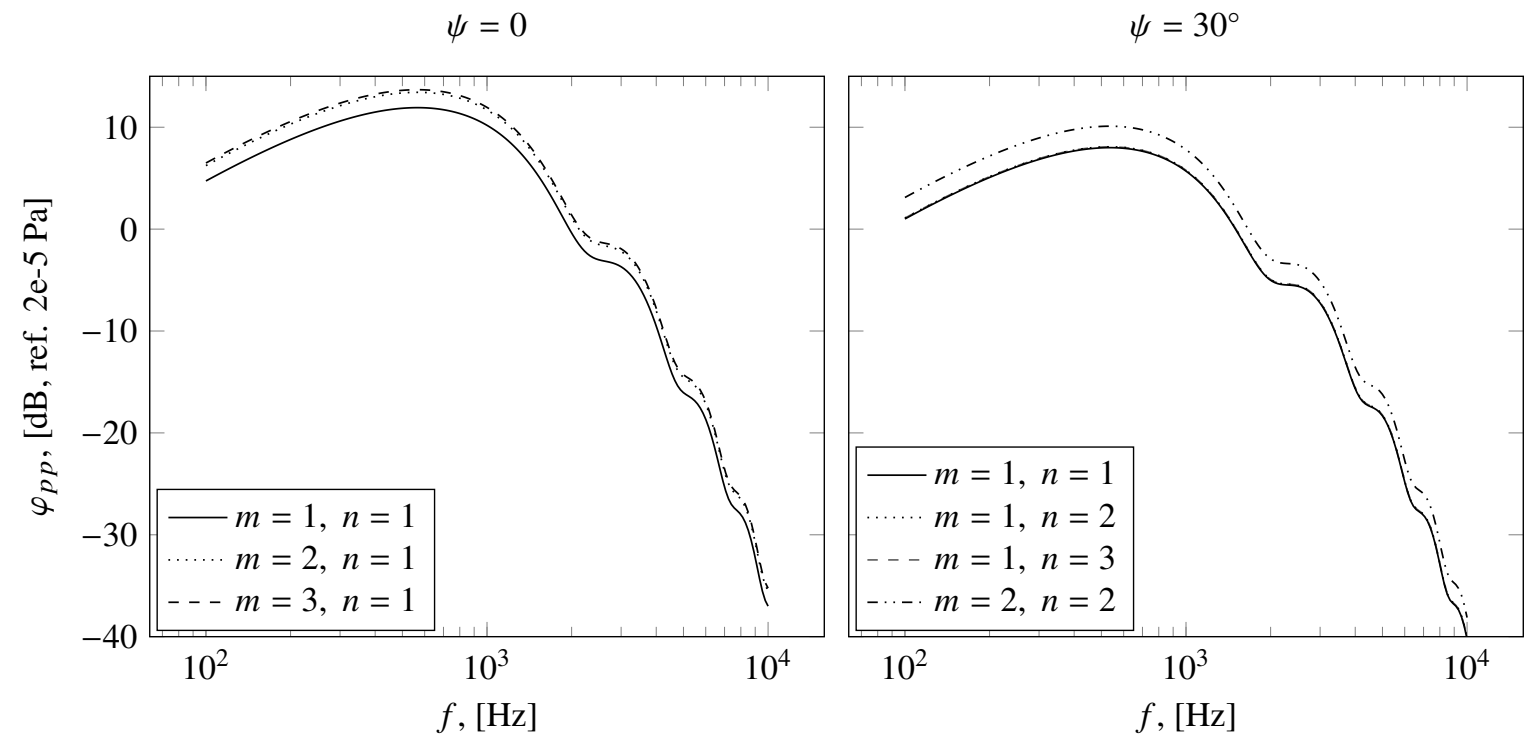

Fig. 7 Effect of the orders of the Butterworth filters on the sound PSD in the midspan plane $\left(x_{2}=0\right)$ at a distance $R=2 \mathrm{~m}$ from the trailing edge.

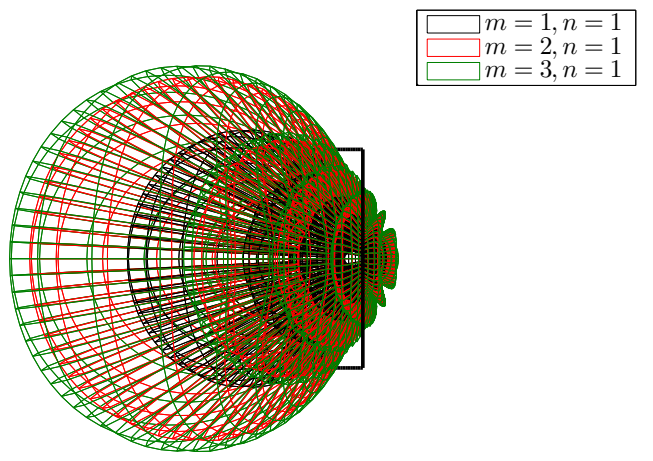

(a) $\psi=0$

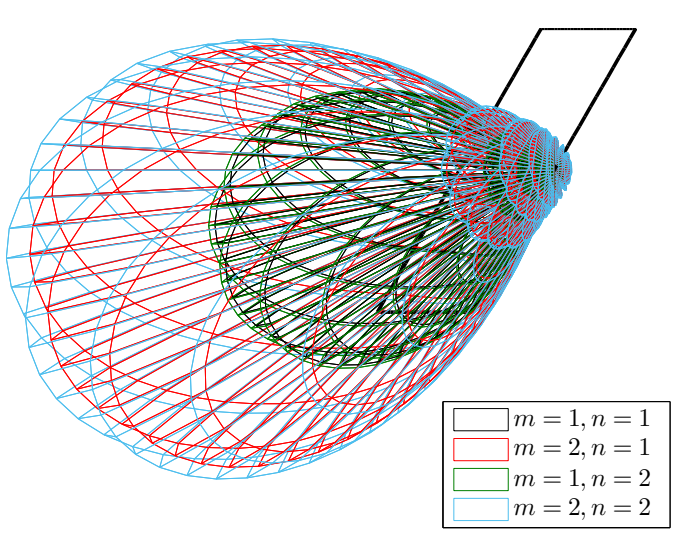

(b) $\psi=30^{\circ}$

Fig. 8 Effect of the order of the Butterworth filters on the directivity of the noise of the CD airfoil on a half-sphere at $f=10 \mathrm{kHz}$. 
and allow assessing the potential for effective noise reduction. Furthermore, the developed model can be efficiently integrated in the early design stage of propellers or fans.

It has been shown that the overall decrease of sound PSD due to sweep could be compensated at high frequency and at some observation angles by the increase of the asymmetry of the directivity pattern. Furthermore, the use of a generalized Corcos' model highlighted the fact that noise predictions can be biased by the hypothesis on the transverse correlation length of wall-pressure fluctuations. More investigation is necessary on this issue, especially in case of rotating blades where the statistics may significantly differ from the stationary airfoil case. This is all the more true since the original model of Corcos is still used for fan applications.

Ongoing research involves the validation of the extension of Amiet's theory to swept airfoils by means of sound measurements in the anechoic chamber of the École Centrale de Lyon.

\section{Acknowledgments}

This project has received funding from the Clean Sky 2 Joint Undertaking under the European Union's Horizon 2020 research and innovation programme under grant agreement No 755543. The Authors also gratefully acknowledge the support of the Canadian NSERC Discovery Grant (no. RGPIN-2014-04111). This work was performed within the framework of the LABEX CeLyA (ANR-10-LABX- 0060) of Université de Lyon, within the program "Investissements d'Avenir' (ANR-16- IDEX-0005) operated by the French National Research Agency (ANR).

\section{References}

[1] Vad, J., "Effects of Sweep and Spanwise Changing Circulation Applies to Airfoils: a Case Study," Journal of Computational and Applied Mechanics, Vol. 5, No. 2, 2004.

[2] Envia, E., and Kerschen, E. J., "Influence of Vane Sweep on Rotor-Stator Interaction Noise,” Tech. rep., NASA, 1990.

[3] Envia, E., and Nallasamy, M., "Design Selection and Analysis of a Swept and Leaned Stator Concept," Tech. rep., NASA, 1998.

[4] de Laborderie, J., and Moreau, S., "Prediction of tonal ducted fan noise," Journal of Sound and Vibration, Vol. 372, 2016 , pp. 105-132. doi:10.1016/j.jsv.2016.02.032.

[5] Woodward, R. P., Elliott, D. M., Hughes, C. E., and Berton, J. J., "Benefits of Swept and Leaned Stators for Fan Noise Reduction," Journal of Aircraft, Vol. 38, No. 6, 2011.

[6] Ommi, F., and Azimi, M., "Main fan noise mitigation technologies in turbofan engines," Aviation, Vol. 18, No. 3, 2014 , pp. 141-146. doi:10.3846/16487788.2014.969881.

[7] Grasso, G., Moreau, S., Christophe, J., and Schram, C., "Multi-disciplinary optimization of a contra-rotating fan," International Journal of Aeroacoustics, Vol. 17, No. 6-8, 2018, pp. 655-686. doi:10.1177/1475472X18789000, URL https://doi .org/ 10.1177/1475472X18789000

[8] Casalino, D., Avallone, F., Gonzalez-Martino, I., and Ragni, D., "Aeroacoustic study of a wavy stator leading edge in a realistic fan/OGV stage," Proceedings of the International Symposium on Transport Phenomena and Dynamics of Rotating Machinery ISROMAC, Maui, Hawaii, 2017.

[9] Bamberger, K., and Carolus, T., "Optimization of Axial Fans with Highly Swept Blades with Respect to Losses and Noise Reduction," Proceedings of the FAN2012 International Conference on Fan Noise, Technology and Numerical Methods, 2012.

[10] Herold, G., Zenger, F., and Sarradj, E., “Influence of blade skew on axial fan component noise,” International Journal of Aeroacoustics, Vol. 16, No. 4-5, 2017, pp. 418-430. doi:10.1177/1475472X17718740.

[11] Krömer, F., and Becker, S., "Experimental investigation of the off-design sound emission of low-pressure axial fans with different fan blade skew," Proceedings of the Euronoise 2018 Conference, Crete., 2018.

[12] Krömer, F. J., Moreau, S., and Becker, S., "Experimental investigation of the interplay between the sound field and the flow field in skewed low-pressure axial fans," Journal of Sound and Vibration, Vol. 442, 2019, pp. 220 - 236. doi:https://doi.org/10. 1016/j.jsv.2018.10.058, URL http://www. sciencedirect.com/science/article/pii/S0022460X18307387.

[13] Amiet, R. K., "Acoustic Radiation from an Airfoil in a Turbulent Stream,” Journal of Sound and Vibration, Vol. 41, No. 4, 1975.

[14] Amiet, R. K., "High Frequency Thin-Airfoil Theory for Subsonic Flow,” AIAA Journal, Vol. 14, No. 8, 1976, pp. $1076-1082$. 
[15] Schwarzschild, K., "Die Beugung und Polarisation des Lichts durch einen Spalt. I.” Mathematische Annalen, Vol. 55, No. 2, 1901, p. 177-247.

[16] Rozenberg, Y., "Modélisation analytique du bruit aérodynamique à large bande des machines tournantes: utilisation de calculs moyennés de mécanique des fluides,” Ph.D. thesis, École Centrale de Lyon, 2007.

[17] Roger, M., and Carazo, A., "Blade-Geometry Considerations in Analytical Gust-Airfoil Interaction Noise Models," 16th AIAA/CEAS Aeroacoustics Conference (31st AIAA Aeroacoustics Conference), Stockholm, SW, 7-9 June, 2010.

[18] Carazo, A., Roger, M., and Omais, M., "Analytical Prediction of Wake-Interaction Noise in Counter-Rotation Open Rotors," Proceedings of the 17th AIAA/CEAS Aeroacoustics Conference (32nd AIAA Aeroacoustics Conference), Portland, OR, June 5 8, 2011.

[19] Roger, M., Schram, C., and Moreau, S., "On vortex-airfoil interaction noise including span-end effects, with application to open-rotor aeroacoustics," Journal of Sound and Vibration, Vol. 333, 2014, pp. 283-306.

[20] Giez, J., Vion, L., Roger, M., and Moreau, S., "Effect of the Edge and Tip Vortex on Airfoil Self Noise and Turbulence Impingement Noise," Proceedings of the 22nd AIAA/CEAS Aeroacoustics Conference, Lyon, FR, May 30 - June 1, 2016.

[21] Quaglia, M. E., Léonard, T., Moreau, S., and Roger, M., "A 3D analytical model for orthogonal blade-vortex interaction noise," Journal of Sound and Vibration, Vol. 399, 2017, p. 104-123.

[22] Adamczyk, J. J., “The passage of an infinite swept airfoil through an oblique gust,” Tech. rep., NASA, 1974.

[23] Amiet, R. K., "Noise Due to Turbulent Flow Past a Trailing Edge," Journal of Sound and Vibration, Vol. 4, No. 3, 1976.

[24] Roger, M., and Moreau, S., "Back-Scattering Correction and Further Extensions of Amiet's Trailing-Edge Noise Model. Part 1: Theory," Journal of Sound and Vibration, Vol. 286, No. 3, 2005, pp. 477-506.

[25] Moreau, S., and Roger, M., "Back-scattering correction and further extensions of Amiet's trailing-edge noise model. Part II: Application," Journal of Sound and Vibration, Vol. 323, No. 1, 2009, pp. 397 - 425. doi:https://doi.org/10.1016/j.jsv.2008.11.051, URL http://wWw.sciencedirect.com/science/article/pii/S0022460X08010079

[26] Lee, S., "Empirical Wall-Pressure Spectral Modeling for Zero and Adverse Pressure Gradient Flows," AIAA Journal, Vol. 56, No. 5, 2018, pp. 1818-1829. doi:10.2514/1.J056528, URL https://doi .org/10.2514/1.J056528

[27] Nodé-Langlois, T., Wlassow, F., Languille, V., Colin, Y., Caruelle, B., Gill, J., Chen, X., Zhang, X., and Parry, A. B., "Prediction of Contra-Rotating Open Rotor broadband noise in isolated and installed configurations," 20th AIAA/CEAS Aeroacoustics Conference, Atlanta, GA, 16-20 June, 2014.

[28] Sanjosé, M., and Moreau, S., "Fast and accurate analytical modeling of broadband noise for a low-speed fan," The Journal of the Acoustical Society of America, Vol. 143, No. 3103, 2018.

[29] Abramowitz, M., and Stegun, I. A., Handbook of Mathematical Functions, Dover Publications, New York, 1970.

[30] Curle, N., and Lighthill, M. J., "The influence of solid boundaries upon aerodynamic sound," Proceedings of the Royal Society of London. Series A. Mathematical and Physical Sciences, Vol. 231, No. 1187, 1955, pp. 505-514. doi:10.1098/rspa.1955.0191, URL https://royalsocietypublishing.org/doi/abs/10.1098/rspa.1955.0191

[31] Roger, M., and Moreau, S., "Addendum to the back-scattering correction of Amiet's trailing-edge noise model," Journal of Sound and Vibration, Vol. 331, 2012, p. 5383-5385.

[32] Amiet, R. K., "Effect of the incident surface pressure field on noise due to turbulent flow past a trailing edge," Journal of Sound and Vibration, Vol. 57, No. 2, 1978, pp. 305-306.

[33] Taylor, G. I., "The spectrum of turbulence," Proceedings of the Royal Society of London. Series A - Mathematical and Physical Sciences, Vol. 164, No. 919, 1938, pp. 476 - 490.

[34] Ffowcs Williams, J. E., and Hall, L. H., "Aerodynamic sound generation by turbulent flow in the vicinity of a scattering half plane," Journal of Fluid Mechanics, Vol. 40, No. 4, 1970, pp. 657-670.

[35] Corcos, G. M., "The structure of the turbulent pressure field in boundary-layer flows," Journal of Fluid Mechanics, Vol. 18, 1964, pp. 353-379. 
[36] Caiazzo, A., D’Amico, R., and Desmet, W., "A Generalized Corcos model for modelling turbulent boundary layer wall pressure fluctuations," Journal of Sound and Vibration, Vol. 372, 2016, pp. 192-210.

[37] Singer, B. A., “Turbulent wall-pressure fluctuations: new model for off-axis cross-spectral density,” Tech. rep., NASA, 1996.

[38] Rozenberg, Y., Robert, G., and Moreau, S., "Wall-pressure spectral model including the adverse pressure gradient effects," AIAA Journal, Vol. 50, No. 10, 2012, pp. 2168-2179.

[39] Moreau, S., and Roger, M., "Effect of Airfoil Aerodynamic Loading on Trailing-Edge Noise Sources," AIAA Journal, Vol. 43, No. 1, 2005, pp. 41-52. doi:10.2514/1.5578.

[40] Christophe, J., Moreau, S., Hamman, C. W., Witteveen, J. A. S., and Iaccarino, G., "Uncertainty Quantification for Trailing-Edge Noise of a Controlled-Diffusion Airfoil," Center for Turbulence Research, Proceedings of the Summer Program, 2010. 